Int. J. Electrochem. Sci., 14 (2019) $6168-6178$

International Journal of

ELECTROCHEMICAL

SCIENCE

$\underline{\text { WwW.electrochemsci.org }}$

\title{
Electrochemical Determination of Acebutolol on the Electrochemically Pretreated Screen Printed Carbon Electrode
}

\author{
Annamalai Yamuna ${ }^{1}$, Periyasamy Sundaresan ${ }^{l}$, Shen-Ming Chen ${ }^{1, *}$, Shaban R. M. Sayed ${ }^{2}$, Tse-Wei \\ Chen ${ }^{1,3}$, Syang-Peng Rwei, ${ }^{3,4}$ Xiaoheng Liu ${ }^{5, *}$ \\ ${ }^{1}$ Department of Chemical Engineering and Biotechnology, National Taipei University of Technology, \\ Taipei 106, Taiwan. \\ ${ }^{2}$ Electron Microscope Unit, Central Laboratory, College of Science, King Saud University, P.O. Box \\ 2455, Riyadh 11451, Saudi Arabia \\ ${ }^{3}$ Research and Development Center for Smart Textile Technology, National Taipei University of \\ Technology, Taipei 106, Taiwan, ROC \\ ${ }^{4}$ Institute of Organic and Polymeric Materials, National Taipei University of Technology, Taipei 106, \\ Taiwan, ROC \\ ${ }^{5}$ Key Laboratory of Education Ministry for Soft Chemistry and Functional Materials, Nanjing \\ University of Science and Technology, Nanjing 210094, China. \\ *E-mail: smchen78@ms15.hinet.net (Shen-Ming Chen), xhliu@ mail.njust.edu.cn (Xiaoheng Liu)
}

doi: $10.20964 / 2019.07 .63$

Received: 9 March 2019 / Accepted: 18 May 2019 / Published: 10 June 2019

This work reports the electrochemical determination of acebutolol (ACE) by the screen printed carbon electrode (SPCE). Before the determination, the SPCE was activated by the electrochemical pretreatment. This pretreatment creates the edge plane active sites on the SPCE surface and also it creates the electrochemically active functional groups. The activation process was confirmed by the Raman, SEM and electrochemical analysis. The activated SPCE (aSPCE) revealed the better electrochemical performance when compared with SPCE. It is presumed that, this remarkable performance was obtained from the edge plane active sites and the electrochemically active functional groups (such as carbonyl groups and hydroxyl groups). Further, this aSPCE utilized to the ACE determination in phosphate buffer solutions which received a great significance of analytical performances. It revealed the good sensitivity $\left(1.02 \mu \mathrm{A} \mathrm{MM}^{-1} \mathrm{~cm}^{-2}\right)$, linear range $(0.01$ to $200 \mu \mathrm{M})$ and low LOD $(0.006 \mu \mathrm{M})$. Moreover, it shows significant anti-interference properties in suspected interfering compounds.

Keywords: Edge plane; polymer inks; beta blocker; heart disease; carbonyl groups and basal plane. 
(C) 2019 The Authors. Published by ESG (www.electrochemsci.org). This article is an open access article distributed under the terms and conditions of the Creative Commons Attribution license (http://creativecommons.org/licenses/by/4.0/). 